\title{
Preoperative diffusion-weighted magnetic resonance imaging and intraoperative frozen sections for predicting the tumor grade in endometrioid endometrial cancer
}

\author{
Tomohito Tanaka ${ }^{1,4}$, Yoshito Terai ${ }^{1,4}$, Satoe Fujiwara ${ }^{1}$, Yoshimichi Tanaka1, Hiroshi \\ Sasaki $^{1}$, Satoshi Tsunetoh ${ }^{1}$, Kazuhiro Yamamoto ${ }^{2,5}$, Takashi Yamada ${ }^{3}$, Yoshifumi \\ Narumi ${ }^{2}$ and Masahide Ohmichi ${ }^{1}$ \\ ${ }^{1}$ Department of Obstetrics and Gynecology, Osaka Medical College, Takatsuki, Japan \\ ${ }^{2}$ Department of Obstetrics and Radiology, Osaka Medical College, Takatsuki, Japan \\ ${ }^{3}$ Department of Obstetrics and Pathology, Osaka Medical College, Takatsuki, Japan \\ ${ }^{4}$ Department of Obstetrics and Gynecology, First Towakai Hospital, Takatsuki, Japan \\ ${ }^{5}$ Department of Obstetrics and Radiology, First Towakai Hospital, Takatsuki, Japan \\ Correspondence to: Tomohito Tanaka, email: gyn123@osaka-med.ac.jp \\ Keywords: endometrial cancer; diffusion weighted image; apparent diffusion coefficient; frozen section; grade \\ Received: February 28, $2018 \quad$ Accepted: November 03, $2018 \quad$ Published: November 27, 2018 \\ Copyright: Tanaka et al. This is an open-access article distributed under the terms of the Creative Commons Attribution License \\ 3.0 (CC BY 3.0), which permits unrestricted use, distribution, and reproduction in any medium, provided the original author and \\ source are credited.
}

\section{ABSTRACT}

Objective: The histological tumor grade is a strong predictor of nodal metastasis in endometrial cancer; as such, an accurate pre- or intraoperative diagnosis is important for performing lymphadenectomy.

Methods: Ninety-one patients with endometrioid endometrial cancer were imaged on DW-MRI with the apparent diffusion coefficient (ADC) calculated and a frozen section (FS) diagnosis made before and at hysterectomy. The diagnostic accuracy for predicting the tumor grade for diffusion weighted magnetic resonance inaging (DWMRI) and the FS diagnosis compared to the ultimate histologic status was analyzed.

Results: Among 91 patients with endometrioid endometrial cancer, high-grade (endometrioid G3) tumors had lower ADC values than low-grade (endometrioid G1/2) tumors. The cut-off of the mean $\mathrm{ADC}_{\text {mean }}$ values for predicting high-grade tumors resulted in $743 \times 10^{-6} \mathrm{~mm}^{2} / \mathrm{sec}$ according to the receiver operating characteristic curve. The true positive rates of ADC values and FSs for the prediction of high-grade tumors did not differ to a statistically significant extent $(73.3 \%$ vs. $66.7 \%, p=0.7)$, however, the true negative rate of ADC values for the prediction of low-grade tumors was significantly lower than that of the FSs $(64.5 \%$ vs. $98.7 \%, p=0.01)$. The kappa statistics of ADC values and FSs were 0.23 and 0.73 , respectively. Of note, all five patients with high-grade tumors for whom intraoperative FSs indicated low-grade tumors were predicted to have high-grade tumors on preoperative DW-MRI.

Conclusion: A FS diagnosis is more suitable for predicting high-grade tumors than DW-MRI; however, physicians should pay close attention to tumors with low ADC values on preoperative DW-MRI.

\section{INTRODUCTION}

The histological tumor grade is an important factor for predicting lymph node metastasis and the prognosis in endometrial cancer. In patients with endometrioid endometrial cancer, the rate of lymph node metastasis was $2.8 \%$ in grade 1 tumors and $5.7 \%$ in grade 2 tumors but increased to $9.4 \%$ in patents with grade 3 tumors 
[1]. Pelvic lymph node dissection (PLND) remains an important surgical procedure for treating endometrial cancer. This procedure is necessary to obtain correct staging and has resulted in a favorable prognosis, especially in patients with high-risk endometrial cancer [2-10], however it may not be needed for low-risk cases, including cases involving low-grade tumors and cases without myometrial invasion. Therefore, the histological tumor grade is an important factor for making decisions regarding surgical procedures, including lymph node dissection. Generally, the tumor grade is determined with a preoperative biopsy [11].

Magnetic resonance imaging (MRI) has been shown to be an accurate imaging technique for the preoperative assessment of endometrial cancer and for evaluating the depth of myometrial invasion [11]. Diffusion-weighted MRI (DW-MRI) is a useful imaging technique for evaluating the Brownian motion of water in tissues. In biological tissues, this motion is restricted by interactions with cell membranes and macromolecules on a microscopic level. Increased tissue cellularity, as seen in tumors, restricts Brownian motion, which can be quantified by the calculation of the apparent diffusion coefficient (ADC).

The purpose of this study was to determine if there is a correlation between the histological tumor grade and $\mathrm{ADC}$ value and to evaluate the diagnostic accuracy of the tumor grade for preoperative DW-MRI and intraoperative frozen sections (FSs) in endometrioid endometrial cancer patients.

\section{RESULTS}

\section{Characteristics of the study participants in test and validation set}

A total of 109 patients with endometrioid endometrial cancer underwent preoperative DW-MRI, preoperative endometrial biopsy and the intraoperative examination of FSs. Ninety-one patients were recruited in the test set. The mean age of the patients was $57.9 \pm$ 10.8 years, and the mean body mass index (BMI) was $23.9 \pm 4.2$. According to the histopathological diagnosis of surgical specimens, a total of $66(72.5 \%)$ patients had International Federation of Gynecology and Obstetrics (FIGO) stage IA disease, 12 (13.2\%) had stage IB disease, $1(1.1 \%)$ had stage II disease, $6(6.6 \%)$ had stage IIIA disease, $3(3.3 \%)$ had stage IIIC disease and $3(3.3 \%)$ had stage IV disease. Histologically, 58 (63.7\%) patients had endometrioid carcinoma of grade 1, $18(19.8 \%)$ had grade 2 , and $15(16.5 \%)$ had grade 3. Eighteen patients were recruited in the validation set. The mean age of the patients was $57.2 \pm 11.3$ years, and the mean BMI was $22.3 \pm 4.8$. A total of $11(61.1 \%)$ patients had FIGO stage IA disease and $7(38.9 \%)$ had stage IB disease. Histologically, 11 $(61.1 \%)$ patients had endometrioid carcinoma of grade 1,5 $(27.8 \%)$ had grade 2 , and $2(11.1 \%)$ had grade 3 (Table 1$)$.

\section{The ADC values for each tumor grade in the test set}

Among the 91 patients in test set, the mean $\mathrm{ADC}_{\text {mean }}$ was $882 \pm 264 \times 10^{-6}\left(\mathrm{~mm}^{2} / \mathrm{sec}\right)$ for grade 1 tumors, 745 $\pm 115 \times 10^{-6}\left(\mathrm{~mm}^{2} / \mathrm{sec}\right)$ for grade 2 tumors and $686 \pm 149$ $\times 10^{-6}\left(\mathrm{~mm}^{2} / \mathrm{sec}\right)$ for grade 3 tumors. The mean $\mathrm{ADC}_{\text {mim }}$ was $737 \pm 224 \times 10^{-6}\left(\mathrm{~mm}^{2} / \mathrm{sec}\right)$ for grade 1 tumors, $640 \pm$ $103 \times 10^{-6}\left(\mathrm{~mm}^{2} / \mathrm{sec}\right)$ for grade 2 tumors and $586 \pm 149 \times$ $10^{-6}\left(\mathrm{~mm}^{2} / \mathrm{sec}\right)$ for grade 3 tumors. The mean $\mathrm{ADC}_{\max }$ was $1048 \pm 306 \times 10^{-6}\left(\mathrm{~mm}^{2} / \mathrm{sec}\right)$ for grade 1 tumors, $883 \pm$ $207 \times 10^{-6}\left(\mathrm{~mm}^{2} / \mathrm{sec}\right)$ for grade 2 tumors and $813 \pm 168 \times$ $10^{-6}\left(\mathrm{~mm}^{2} / \mathrm{sec}\right)$ for grade 3 tumors. Each of the three ADC values was significantly higher for grade 1 tumors than for grade 3 tumors. In contrast, the ADC values were not different significantly between grade 1 and 2 tumors and between grade 2 and 3 tumors (Figure 1).

\section{The diagnostic accuracy of the ADC values, preoperative biopsy and intraoperative FSs in predicting high-grade tumors in the test set}

Among 91 patients in test set, the mean $\mathrm{ADC}_{\text {mean }}$, $\mathrm{ADC}_{\text {min }}$ and $\mathrm{ADC}_{\text {max }}$ were significantly lower in the highgrade tumors than in the low-grade tumors $\left(\mathrm{ADC}_{\text {mean }}, 686\right.$ \pm 149 vs. $850 \pm 244 \times 10^{-6} \mathrm{~mm}^{2} / \mathrm{sec}, \mathrm{p}=0.01 ; \mathrm{ADC}_{\text {min }}, 586$ \pm 149 vs. $714 \pm 206 \times 10^{-6} \mathrm{~mm}^{2} / \mathrm{sec}, \mathrm{p}=0.02 ; \mathrm{ADC}_{\max }, 1009$ \pm 293 vs. $814 \pm 168 \times 10^{-6} \mathrm{~mm}^{2} / \mathrm{sec}, \mathrm{p}=0.01$ ) (Figure 2). The area under the curve (AUC) values of the $\mathrm{ADC}_{\text {mean }}$, $\mathrm{ADC}_{\text {min }}$ and $\mathrm{ADC}_{\text {max }}$ for predicting high-grade tumors were $0.71,0.68$ and 0.72 , respectively. Figure 3 shows the receiver operating characteristic curves (ROCs). The cutoff values of $\mathrm{ADC}_{\text {mean }}, \mathrm{ADC}_{\text {min }}$ and $\mathrm{ADC}_{\text {max }}$ for predicting high-grade tumors were 743,603 and $877 \times 10^{-6}\left(\mathrm{~mm}^{2} /\right.$ sec), respectively.

The true positive rates of $\mathrm{ADC}_{\text {mean }}, \mathrm{ADC}_{\text {min }}, \mathrm{ADC}_{\text {max }}$, preoperative biopsy, and FS in the diagnosis of high-grade rumors were $73.3 \%, 66.7 \%, 73.3 \%, 40.0 \%$ and $66.7 \%$, respectively. The true negative rates of $\mathrm{ADC}_{\text {mean }}, \mathrm{ADC}_{\text {min }}$, $\mathrm{ADC}_{\text {max }}$, preoperative biopsy, and FS in the diagnosis of low-grade tumors were $64.5 \%, 69.7 \%, 69.7 \%, 89.0 \%$ and $98.7 \%$, respectively. The kappa statistics $(95 \%$ confidence interval) of $\mathrm{ADC}_{\text {mean }}, \mathrm{ADC}_{\text {min }}, \mathrm{ADC}_{\max }$, preoperative biopsy, and FS were 0.23 (0.06-0.41), 0.25 (0.05-0.44), 0.29 (0.10-0.47), 0.43 (0.17-0.69) and 0.73 (0.53-0.93), respectively (Table 2 ). The true positive rates of combined methods in the prediction of high-grade tumors were as follows: preoperative biopsy and FS, 73.3\%; FS and DW-MRI, 100\%; and preoperative biopsy and DW-MRI, $80 \%$. Although the combination of FS and DW-MRI was had a true positive rate of $100 \%$, the true negative rate of this combination in the prediction of low-grade tumors was $63.2 \%$ with a kappa statistic of 0.36 . Thus, the combination of FS and DW-MRI had an excellent true positive rate for the prediction high-grade tumors, but was associated with a high rate of false positive results. Of 
Table 1: Characteristics of patients with endometrial cancer who underwent pre-operative DW-MRI and intraoperative frozen section analyses

\begin{tabular}{|c|c|c|}
\hline Characteristic & Test set $(\%)$ & Validation set $(\%)$ \\
\hline Number of patients & 91 & 18 \\
\hline Age, years old ${ }^{*}$ & $57.9 \pm 10.8$ & $57.2 \pm 11.3$ \\
\hline BMI, $\mathrm{kg} / \mathrm{m}^{2^{*}}$ & $23.9 \pm 4.2$ & $22.3 \pm 4.8$ \\
\hline \multicolumn{3}{|l|}{ FIGO stage } \\
\hline IA & $66(72.5)$ & $11(61.1)$ \\
\hline IB & $12(13.2)$ & $7(38.9)$ \\
\hline II & $1(1.1)$ & 0 \\
\hline IIIA & $6(6.6)$ & 0 \\
\hline IIIC & $3(3.3)$ & 0 \\
\hline IV & $3(3.3)$ & 0 \\
\hline \multicolumn{3}{|l|}{ Grade } \\
\hline 1 & $58(63.7)$ & $11(61.1)$ \\
\hline 2 & $18(19.8)$ & $5(27.8)$ \\
\hline 3 & $15(16.5)$ & $2(11.1)$ \\
\hline
\end{tabular}

*Based on an ANOVA (mean \pm SD)

Abbreviations: BMI, body mass index; FIGO, federation of Gynecology and Obstetrics.

note, all five patients with high-grade tumors for whom intraoperative frozen sections indicated low-grade tumors were predicted to have high-grade tumors on preoperative DW-MRI. On preoperative endometrial biopsy, the diagnoses of these 5 cases were endometrioid carcinoma G1 $(n=2), G 2(n=2)$ and G3 $(n=1)$.

\section{The ADC values of low-grade (grade 1/2) and high-grade (grade 3) tumors in the validation set}

When the cutoff value in test set was applied to the 18 patients in validation set, the true positive rates of the $\mathrm{ADC}_{\text {mean }}, \mathrm{ADC}_{\text {min }}$ and $\mathrm{ADC}_{\text {max }}$ in the prediction of highgrade tumors were $100 \%, 50.0 \%$ and $50.0 \%$, respectively. The true negative rates of the $\mathrm{ADC}_{\text {mean }}, \mathrm{ADC}_{\text {min }}$, and $\mathrm{ADC}_{\text {max }}$ in the prediction of low-grade tumors were $68.8 \%$, $75.0 \%$ and $81.3 \%$, respectively. The kappa statistics of the $\mathrm{ADC}_{\text {mean }}, \mathrm{ADC}_{\text {min }}$ and $\mathrm{ADC}_{\text {max }}$ were 0.33, 0.15 and 0.22, respectively (Table 3 ).

\section{DISCUSSION}

In the current study, the true positive rates of the ADC values and FSs in the prediction of high-grade tumors did not differ to a statistically significant extent; however, the true negative rate of the ADC values in the prediction of low-grade tumors was significantly lower than that of FSs. The kappa statistics of the ADC value and
FS were 0.23 and 0.73 , respectively. Although FSs were found to be more useful for predicting high-grade tumors than DW-MRI, all five patients with high-grade tumors for whom intraoperative frozen sections indicated lowgrade tumors were predicted to have high-grade tumors on preoperative DW-MRI. Therefore, careful management should be performed in cases of endometrioid tumors with low ADC values.

While the ADC values of endometrial cancer have been evaluated, the relationship between the ADC values and histological grade remains controversial. Several authors found no correlation with the tumor grade [12-14], whereas more recent studies have shown that high-grade tumors have lower ADC values than low-grade tumors [15-17]. Rechichi et al. reported a prospective study of ADC values in endometrial cancer. Among 106 patients, including 70 endometrial cancer patients and 36 control subjects with normal endometrium, the mean ADC value of endometrial cancer tissue was significantly lower than that of normal endometrium; however, there was no significant difference in the ADC values between the histological grade. In this study, the mean ADC values $\left(\times 10^{-6} \mathrm{~mm}^{2} /\right.$ sec) of grade 1, 2 and 3 tumors were $790 \pm 8,760 \pm 14$ and $750 \pm 12$, respectively [12]. Bharwani et al. reported the ADC values in 42 endometrial lesions, including 23 endometrial cancers and 19 benign lesions. Although the mean ADC value for endometrial carcinoma tissue was significantly lower than that of benign endometrial 
tissue, there were no statistically significant differences between the tumor grades. The cut-off of the mean ADC values for predicting malignancy was $<1280 \times 10^{-6}\left(\mathrm{~mm}^{2} /\right.$ sec). In this setting, the sensitivity and specificity for malignancy were $87 \%$ and $100 \%$, respectively. The mean and minimum ADC values $\left(\times 10^{-6} \mathrm{~mm}^{2} / \mathrm{sec}\right)$ for each histological grade were $1020 \pm 290$ and $740 \pm 240$ (grade 1), $880 \pm 390$ and $640 \pm 360$ (grade 2) and $940 \pm 320$ and $720 \pm 360$ (grade 3), respectively [13]. Kishimoto et al. showed that the ADC values were significantly inversely correlated with the tumor cellularity. However, no significant relationship was observed between the $\mathrm{ADC}$ values and tumor grade. The mean $\mathrm{ADC}$ values $\left(\times 10^{-6} \mathrm{~mm}^{2} / \mathrm{sec}\right)$ of grade 1,2 and 3 tumors were $880 \pm$ $265,800 \pm 178$ and $810 \pm 117$, respectively [14]. Woo et al. reported a histogram analysis of ADC values based on the entire tumor volume to determine the grade of endometrial cancer in 33 patients. The standard deviation, quartile and $75^{\text {th }}, 90^{\text {th }}$ and $95^{\text {th }}$ percentiles of ADC showed significant differences between grades and between high and low grades. An ROC curve analysis yielded sensitivities and specificities of $75 \%$ and $96 \%, 62.5 \%$ and $92 \%, 100 \%$ and $52 \%, 100 \%$ and $72 \%$, and $100 \%$ and $80 \%$, using the standard deviation, quartiles and $75^{\text {th }}, 90^{\text {th }}$ and $95^{\text {th }}$ percentiles to identify high-grade tumors with corresponding areas under the curve of 787, 792, 765880 and $925 \times 10^{-6}\left(\mathrm{~mm}^{2} / \mathrm{sec}\right)$, respectively [15]. Nougaret et al. showed that the minimum $10^{\text {th }}, 25^{\text {th }}, 50^{\text {th }}, 75^{\text {th }}$ and $90^{\text {th }}$ percentile ADC values were significantly lower in grade 3 tumors than in grade 1 and 2 tumors among 97 endometrial cancer patients. For the medium ADC values according to the ROC curve results, an ADC value lower than $730 \times 10^{-6}\left(\mathrm{~mm}^{2} / \mathrm{sec}\right)$ was associated with grade 3 tumors with a sensitivity of $77 \%$ and specificity of $75 \%$ [16]. Yan et al. reported the ADC values in 98 endometrial cancer patients. The mean ADC values for high-grade endometrioid adenocarcinomas were significantly lower than those for low-grade tumors (800 vs. $962 \times 10^{-6}\left[\mathrm{~mm}^{2} /\right.$ $\mathrm{sec}$, $\mathrm{p}=0.002$ ). However, no significant differences were noted in mean and minimum ADC values among tumor grades [17]. Although the relationship between the ADC values and histological tumor grade remains controversial, grade 3 tumors have lower ADC value than grade 1 tumors in many published literatures. In the current study, the ADC values were significantly lower for grade 3 tumors than for grade 1 tumors.

In contrast, it has been reported that intraoperative FS diagnoses tend to agree with the final pathology [11,

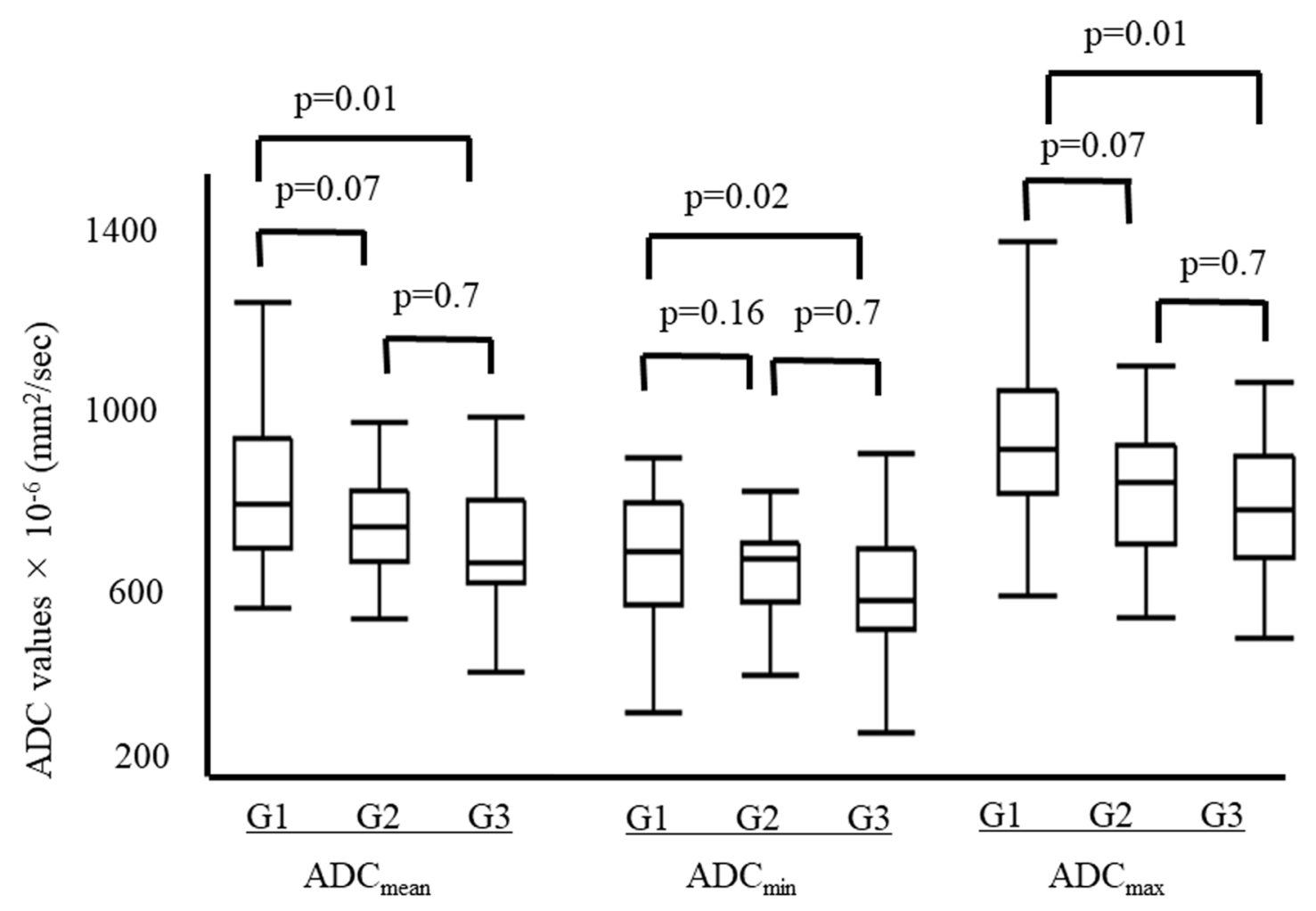

Figure 1: The ADC values for each tumor grade. The mean $\mathrm{ADC}_{\text {mean }}$ was $882 \pm 264 \times 10^{-6}\left(\mathrm{~mm}^{2} / \mathrm{sec}\right)$ for grade 1 tumors, $745 \pm 115$ $\times 10^{-6}\left(\mathrm{~mm}^{2} / \mathrm{sec}\right)$ for grade 2 tumors and $686 \pm 149 \times 10^{-6}\left(\mathrm{~mm}^{2} / \mathrm{sec}\right)$ for grade 3 tumors. The mean $\mathrm{ADC}_{\min }$ was $737 \pm 224 \times 10^{-6}\left(\mathrm{~mm}^{2} / \mathrm{sec}\right)$ for grade 1 tumors, $640 \pm 103 \times 10^{-6}\left(\mathrm{~mm}^{2} / \mathrm{sec}\right)$ for grade 2 tumors and $586 \pm 149 \times 10^{-6}\left(\mathrm{~mm}^{2} / \mathrm{sec}\right)$ for grade 3 tumors. The mean $\mathrm{ADC}_{\max }$ was $1048 \pm 306 \times 10^{-6}\left(\mathrm{~mm}^{2} / \mathrm{sec}\right)$ for grade 1 tumors, $883 \pm 207 \times 10^{-6}\left(\mathrm{~mm}^{2} / \mathrm{sec}\right)$ for grade 2 tumors and $813 \pm 168 \times 10^{-6}\left(\mathrm{~mm}^{2} / \mathrm{sec}\right)$ for grade 3 tumors. Each of the mean ADC values was significantly higher for grade 1 tumors than for grade 3 tumors. In contrast, none of the mean ADC values differed significantly between grade 1 and 2 tumors and grade 2 and 3 tumors. 
18, 19]. Karabagli et al. reported that the agreement for tumor grade between FSs and PSs was $89.9 \%$, with a kappa value of 0.84 [18]. Stephan et al. reported that the histological grade evaluation based on an FS had $98 \%$ sensitivity and $53 \%$ specificity compared to PSs. There were no discrepancies in the FS assessment of high-grade tumors, with all confirmed by PSs [19]. We previously reported on the histological agreement between FSs and PSs in endometrial cancer. When grade 3 endometrioid carcinoma, serous carcinoma, clear cell carcinoma and carcinosarcoma were considered high-grade tumors, the grade evaluation at the time of obtaining an FS had $70.2 \%$ sensitivity and $99.0 \%$ specificity, with a kappa value of $0.75[11]$.

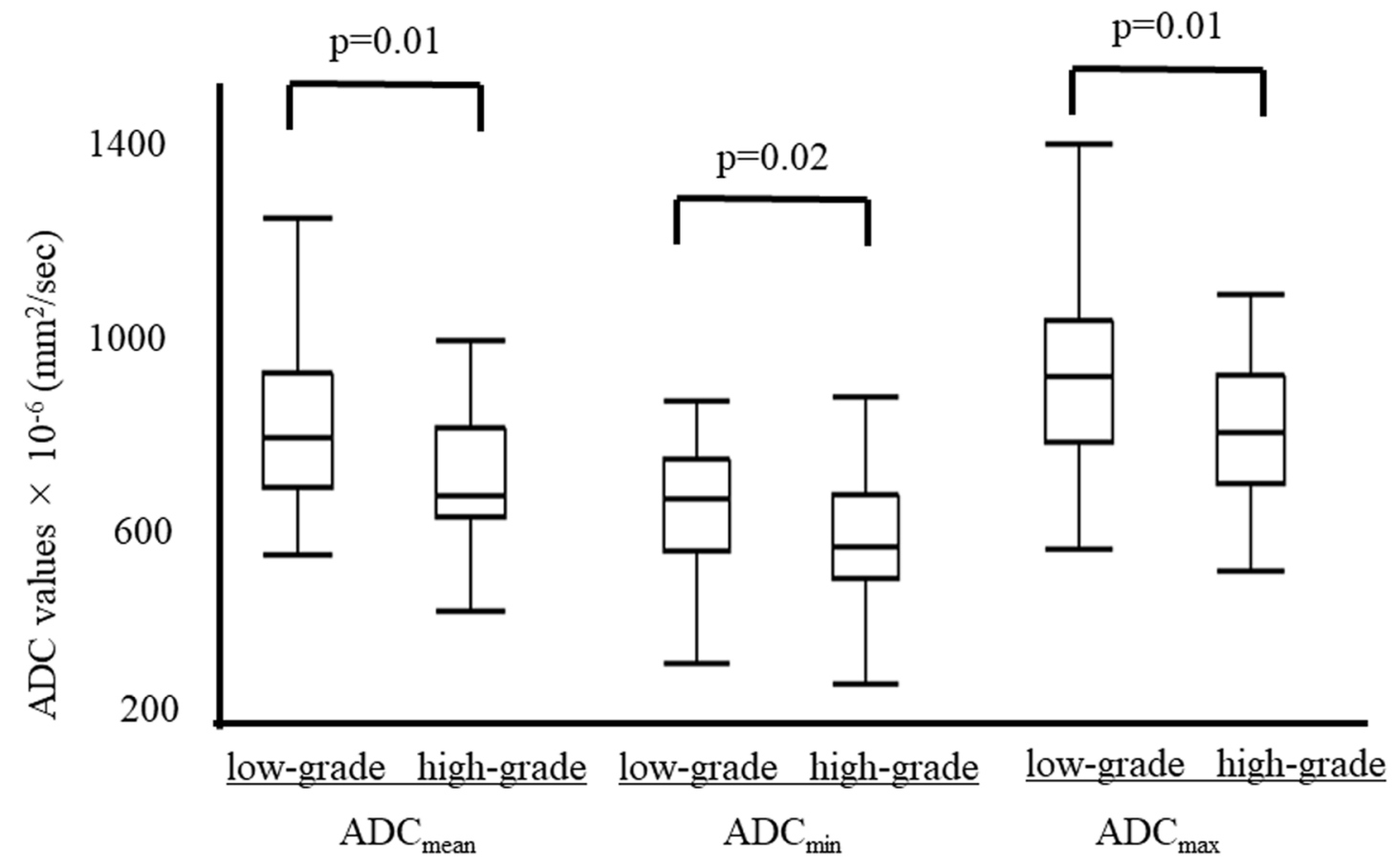

Figure 2: The ADC values compared between low-grade (grade 1/2) and high-grade (grade 3) tumors. The mean $A D C$ mean, $\mathrm{ADC}_{\min }$ and $\mathrm{ADC}_{\text {max }}$ were significantly lower in high-grade tumors than in low-grade tumors $\left(\mathrm{ADC}_{\text {mean }}, 686 \pm 149 \mathrm{vs} .850 \pm 244 \times 10^{-6} \mathrm{~mm}^{2} /\right.$ $\mathrm{sec}, \mathrm{p}=0.01 ; \mathrm{ADC}_{\min }, 586 \pm 149$ vs. $\left.714 \pm 206 \times 10^{-6} \mathrm{~mm}^{2} / \mathrm{sec}, \mathrm{p}=0.02 ; \mathrm{ADC}_{\max }, 1009 \pm 293 \mathrm{vs} .814 \pm 168 \times 10^{-6} \mathrm{~mm}^{2} / \mathrm{sec}, \mathrm{p}=0.01\right)$.
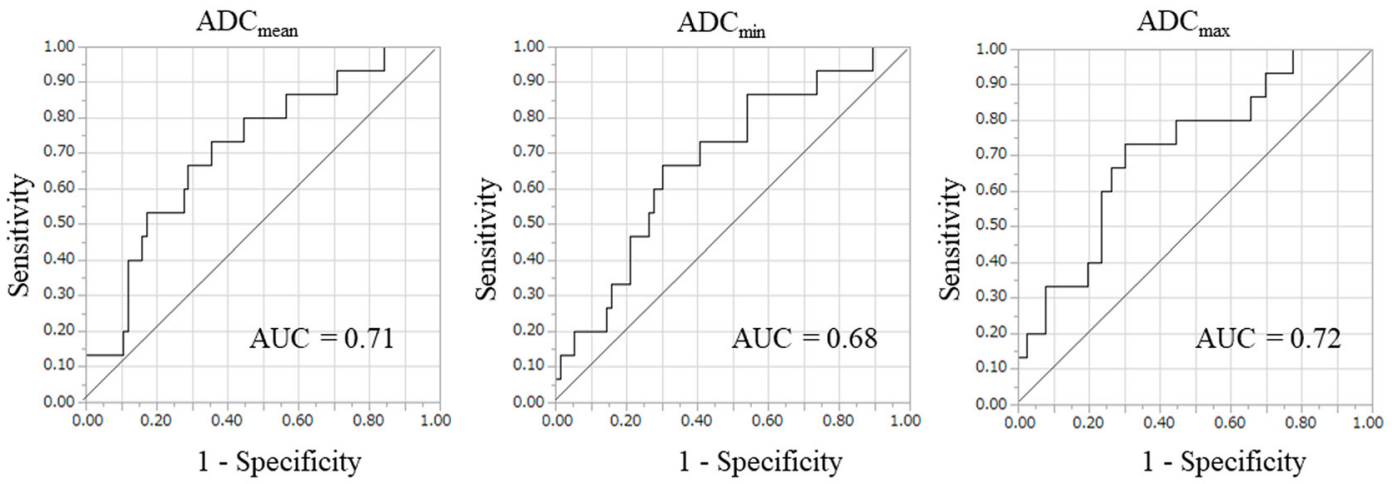

Figure 3: The receiver operating characteristic curve (ROC) for predicting high-grade tumors. The area under curve (AUC) values of the $\mathrm{ADC}_{\text {mean }}, \mathrm{ADC}_{\text {min }}$ and $\mathrm{ADC}_{\max }$ for predicting high-grade tumors were $0.71,0.68$ and 0.72 , respectively. According to $\mathrm{ROC}$ analysis, the cut-off values of the $\mathrm{ADC}_{\text {mean }}, \mathrm{ADC}_{\min }$ and $\mathrm{ADC}_{\max }$ for predicting high-grade tumors were 743,603 and $877 \times 10^{-6}$ $\left(\mathrm{mm}^{2} / \mathrm{s}\right)$, respectively. 
Table 2: The correlation of the tumor grade with the preoperative MRI parameters, and the preoperative biopsy, intraoperative frozen section and final paraffin section findings in the test set

\begin{tabular}{|c|c|c|c|c|c|c|c|c|c|c|c|}
\hline & \multicolumn{2}{|c|}{ ADCmean } & \multicolumn{2}{|c|}{ ADCmin } & \multicolumn{2}{|c|}{ ADCmax } & \multicolumn{2}{|c|}{ Biopsy } & \multicolumn{2}{|c|}{ FS } & \multirow[t]{2}{*}{ PS } \\
\hline & $\begin{array}{c}\text { G1 or } \\
\text { G2 }\end{array}$ & G3 & $\begin{array}{c}\text { G1 or } \\
\text { G2 }\end{array}$ & G3 & $\begin{array}{c}\text { G1 or } \\
\text { G2 }\end{array}$ & G3 & $\begin{array}{c}\text { G1 or } \\
\text { G2 }\end{array}$ & G3 & $\begin{array}{c}\text { G1 or } \\
\text { G2 }\end{array}$ & G3 & \\
\hline $\begin{array}{l}\text { G1 or } \\
\text { G2 }\end{array}$ & 49 & 27 & 53 & 23 & 53 & 23 & 73 & 3 & 75 & 1 & $76(83.5)$ \\
\hline G3 & 4 & 11 & 5 & 10 & 4 & 11 & 9 & 6 & 5 & 10 & $15(16.5)$ \\
\hline Total & 53 & 38 & 58 & 33 & 57 & 34 & 82 & 9 & 80 & 11 & 91 \\
\hline $\begin{array}{l}\text { True } \\
\text { positive } \\
\text { high- } \\
\text { grade } \\
\text { tumors }\end{array}$ & \multicolumn{2}{|c|}{$73.3 \%$} & \multicolumn{2}{|c|}{$66.7 \%$} & \multicolumn{2}{|c|}{$73.3 \%$} & \multicolumn{2}{|c|}{$40.0 \%$} & \multicolumn{2}{|c|}{$66.7 \%$} & \\
\hline $\begin{array}{l}\text { True } \\
\text { negative } \\
\text { low- } \\
\text { grade } \\
\text { tumors }\end{array}$ & \multicolumn{2}{|c|}{$64.5 \%$} & \multicolumn{2}{|c|}{$69.7 \%$} & \multicolumn{2}{|c|}{$69.7 \%$} & \multicolumn{2}{|c|}{$89.0 \%$} & \multicolumn{2}{|c|}{$98.7 \%$} & \\
\hline $\begin{array}{l}\text { Kappa } \\
\text { statistics } \\
(95 \% \\
\text { CI) }\end{array}$ & \multicolumn{2}{|c|}{$0.23(0.06-0.41)$} & \multicolumn{2}{|c|}{$0.25(0.05-0.44)$} & \multicolumn{2}{|c|}{$0.29(0.10-0.47)$} & \multicolumn{2}{|c|}{$0.43(0.17-0.69)$} & \multicolumn{2}{|c|}{$0.73(0.53-0.93)$} & \\
\hline
\end{tabular}

Abbreviations: ADC, apparent diffusion coefficient; FS, frozen section; PS, paraffin section.

Table 3: The correlation of the tumor grade with the preoperative MRI parameters and the final paraffin section findings in the validation set

\begin{tabular}{|c|c|c|c|c|c|c|c|}
\hline & \multicolumn{2}{|c|}{ ADCmean } & \multicolumn{2}{|c|}{ ADCmin } & \multicolumn{2}{|c|}{ ADCmax } & \multirow[t]{2}{*}{ PS } \\
\hline & G1 or G2 & G3 & G1 or G2 & G3 & G1 or G2 & G3 & \\
\hline G1 or G2 & 11 & 5 & 12 & 4 & 13 & 3 & $16(88.9)$ \\
\hline G3 & 0 & 2 & 1 & 1 & 1 & 1 & $2(11.1)$ \\
\hline Total & 11 & 7 & 13 & 5 & 14 & 4 & 18 \\
\hline $\begin{array}{l}\text { True positive } \\
\text { high-grade } \\
\text { tumors }\end{array}$ & \multicolumn{2}{|c|}{$100 \%$} & \multicolumn{2}{|c|}{$50.0 \%$} & \multicolumn{2}{|c|}{$50.0 \%$} & \\
\hline $\begin{array}{l}\text { True } \\
\text { negative } \\
\text { low-grade } \\
\text { tumors }\end{array}$ & \multicolumn{2}{|c|}{$68.8 \%$} & \multicolumn{2}{|c|}{$75.0 \%$} & \multicolumn{2}{|c|}{$81.3 \%$} & \\
\hline $\begin{array}{l}\text { Kappa } \\
\text { statistics }\end{array}$ & \multicolumn{2}{|c|}{0.33} & \multicolumn{2}{|c|}{0.15} & \multicolumn{2}{|c|}{0.22} & \\
\hline
\end{tabular}

Abbreviations: ADC, apparent diffusion coefficient; FS, frozen section; PS, paraffin section.

The present study is associated with three major limitations that may reduce its value. First, the sample size was quite small for assessing the apparent ADC values. Second, the same pathologists performed both the FS and
PS evaluations; this may have caused some bias in favor of the FS diagnosis. Third, the ADC values differed among tumor sites. Fourth, the fact that the whole tumor was not defined and that the $\mathrm{ADC}_{\text {mean }}, \mathrm{ADC}_{\text {min }}$ and $\mathrm{ADC}_{\text {max }}$ were 

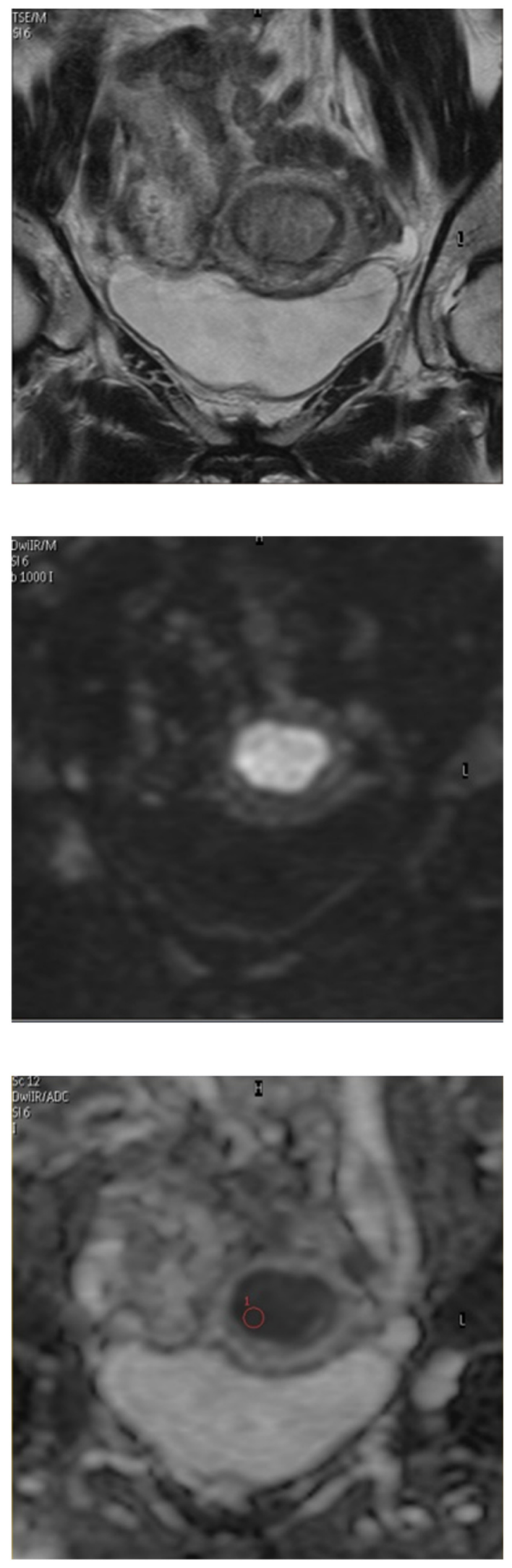

Figure 4: Apparent diffusion coefficient (ADC) analyses of endometrial lesions. MRI identified a endometrial carcinoma invading the endometrial cavity with intermediate signal intensity on axial oblique $\mathrm{T} 2$-weighted imaging (A) and high signal intensity on diffusion weighted imaging (DWI) (B). A region of interest $\left(59.3 \mathrm{~mm}^{2}\right)$ was manually defined within the smallest ADC area of the endometrial lesion on a DW-ADC image (C). 
reported might have influenced the outcome. As such, our results must be confirmed in further studies.

In conclusion, true positive rates of $\mathrm{ADC}$ values and frozen sections in the prediction of high-grade tumors did not differ to a statistically significant extent, however, the true negative rate of the ADC values in the prediction of low-grade tumors was significantly lower than that of FSs; DW-MRI had an excellent true positive rate for the prediction high-grade tumors, but was associated with a high rate of false positive results. Although FSs were found to be more useful for predicting high-grade tumors than DW-MRI, all five patients with high-grade tumors for whom intraoperative frozen sections indicated lowgrade tumors were predicted to have high-grade tumors on preoperative DW-MRI. Given these findings, careful management should be performed for endometrioid tumors with low ADC values.

\section{MATERIALS AND METHODS}

\section{Participants}

Between September 2014 and July 2018, a total of 109 patients with a diagnosis based on preoperative endometrial curettage, preoperative MRI and intraoperative FSs underwent hysterectomy for endometrial cancer at Osaka Medical College in Japan. Patients who met the following criteria were eligible for inclusion in the study: (1) underwent preoperative DW-MRI with ADC map at First Towakai hospital by the same radiologist with the same imaging systems; (2) underwent laparoscopic or abdominal hysterectomy with bilateral salpingo-oophorectomy at Osaka Medical College; (3) received an intraoperative FS diagnosis; (4) did not receive any treatment, including chemotherapy and radiotherapy, before surgery. Ninety-one patients who were treated between September 2014 and September 2017 were assigned to the test set and 18 patients treated between October 2017 and July 2018 were assigned to the validation set.

After obtaining Institutional Review Board approval, the medical records were retrospectively reviewed. The ADC values on DW-MRI were evaluated in each tumor grade and the final determinations were made based on the findings of evaluations using paraffin sections (PSs). The histological grades determined based on preoperative DW-MRI or FS findings and on PS were also assessed.

\section{MRI}

All pelvic MR images were obtained using a 1.5-T MR imaging unit (Philips Healthcare, Best, The Netherlands) within a month before surgery. Routine pelvic MRI scans were acquired as Supplementary Table 1. Axial oblique scans were obtained perpendicular to the endometrial cavity, resulting in a short-axis view.
Dynamic contrast material-enhanced (DCE) imaging of the pelvis was performed after the administration of 0.1 $\mathrm{mmol} / \mathrm{kg}$ of body weight gadolinium chelate (Gadovist; Bayer, Mississauga, Ontario, Canada) using 3-dimensional gradient-echo T1 weighted SPAIR with the parameters in Supplementary Table 1 . Images were acquired at multiple phases of contrast medium enhancement in both the sagittal and axial oblique planes at 30,60, 90 and 180 seconds.

\section{ADC analyses of endometrial lesions}

ADC maps were generated on the scanner console using axial oblique DW $\mathrm{b}=1000$ and $\mathrm{b}=0$ images. For each patient, a region of interest (ROI: 11.9-59.3 $\mathrm{mm}^{2}$; mean $49.2 \pm 16.9 \mathrm{~mm}^{2}$ ) was manually defined by two radiologists (with 30 years of experience in gynecological MRI) to be as large as possible (max $59.3 \mathrm{~mm}^{2}$ ) within the smallest ADC area of endometrial lesion visualized on DW imaging $(b=0)$. Care was taken to avoid contamination of this ROI by adjacent normal endometrium or myometrium or by areas of fluid/necrosis within the endometrial cavity (Figure 4). The ROI was determined after discussion between two radiologists. The mean, minimum and maximum ADC values for each tumor grade were compared using a one-way analysis of variance (ANOVA). A ROC curve was generated to evaluate the diagnostic accuracy with the tumor grade as a predictor for DWI. The cut-off point for the ROC was predetermined by JMP software in order to automatically minimize the mathematical distance between the ROC curve and the ideal point, as a method to minimize any misclassification of the tumor grade. The $\mathrm{ADC}_{\text {mean }}, \mathrm{ADC}_{\text {min }}$ and $\mathrm{ADC}_{\text {max }}\left(\mathrm{mm}^{2} / \mathrm{sec}\right)$ were calculated using the software program for the Philips workstation (Extended MR Workspace; Philips Healthcare, Best, The Netherlands).

\section{Histopathology}

We performed the FS analysis using the following procedure: After hysterectomy, the uterine anterior wall was sectioned through the cervical canal and the endometrial cavity longitudinally and then sectioned horizontally from the fundus to the ostium of the fallopian tube. Gynecologists assessed the deepest point of invasion and oversaw the selection of sections for freezing. One full-thickness section of an endometrial tumor, grossly assessed to be deepest point of invasion, with underlying myometrium and serosa was frozen in Optimal Cutting Temperature media and sectioned on a cryostat into 5 - $\mu \mathrm{m}$ slices. Five different levels were cut, separated by approximately $25-50 \mu \mathrm{m}$, which were then stained with rapid hematoxylin and eosin staining for microscopy.

Both the microscopic depth of myometrial invasion as well as the tumor subtype and grade were determined on the single frozen section. Occasionally, 
an additional section was frozen when the first slides showed equivocal findings or the sections were of a particularly poor quality. The microscopic diagnosis was performed by two pathologists. In most cases, the frozen section remnant was then submitted for permanent histology, and several additional sections of tumor were paraffin-embedded and used for the final microscopic analysis. The same pathologists verified the final pathology report. When the pathologists made a different diagnosis, discussions were held until a consensus was reached.

\section{True positive high-grade tumors and true negative low-grade tumors}

The true positive rate of high-grade tumors was defined as the total number of high-grade tumors on preoperative DW-MRI or intra-operative FSs divided by the total number of high-grade tumors on final pathology. The true negative rate of low-grade tumors was defined as the total number of low-grade tumors on pre-operative DWMRI or intra-operative FSs divided by the total number of low-grade tumors on final pathology.

\section{Statistical analyses}

All of the statistical analyses were performed using the JMP software package (version. 13.1.0; SAS institution Japan, Tokyo, Japan). The diagnostic agreement between the DWI, preoperative biopsy or FS findings and the final pathology was calculated using kappa statistics. Continuous variables are expressed as the mean \pm standard deviation (SD). The Mann-Whitney U-test was used to compare continuous variables between the two groups. Tukey's honestly significant difference (HSD) was used for making multiple comparisons in datasets with continuous variables containing more than two groups. Fisher's exact test was used to compare frequencies. $\mathrm{P}$ values of $<0.05$ were considered to indicate statistical significance.

\section{Abbreviations}

ADC: apparent diffusion coefficient; FS: frozen section; FIGO: International Federation of Gynecology and Obstetrics; DW-MRI: diffusion-weighted magnetic resonance imaging; PLND: pelvic lymph node dissection; ADC: apparent diffusion coefficient; BMI: body mass index; AUC: area under the curve; ROC: receiver operating characteristic; PS: paraffin sections; TSE: turbo spin echo; SPAIR: spectral attenuated inversion recovery; VISTA: volume isotropic TSE acquisition; 3DG: 3-dimensional gradient-echo; TR: repetition time; TE: echo time; FOV: field of view; DCE: Dynamic contrast material-enhanced; ROI: region of interest; ANOVA: analysis of variance; SD: standard deviation; HSD: honestly significant difference.

\section{Author contributions}

T.T., and M.O. designed study; T.T., Y.Te., S.F., Y.Ta. and H.S. performed data collection and analysis; T.T., Y.Te, Y.Ta., and T.Y. made diagnosis of histological analyses; T.T., S.T., K.Y. and Y.N. made diagnosis of image analyses; T.T., and M.O. wrote the paper.

\section{CONFLICTS OF INTEREST} interest.

The authors declare that they have no conflicts of

\section{FUNDING}

The authors have received no funding for this article.

\section{REFERENCES}

1. Vargas R, Rauh-Hain JA, Clemmer J, Clark RM, Goodman A, Growdon WB, Schorge JO, Del Carmen MG, Horowitz NS, Boruta DM 2nd. Tumor size, depth of invasion, and histologic grade as prognostic factors of lymph node involvement in endometrial cancer: a SEER analysis. Gynecol Oncol. 2014; 133: 216-20. https://doi. org/10.1016/j.ygyno.2014.02.011.

2. Kilgore LC, Partridge EE, Alvarez RD, Austin JM, Shingleton HM, Noojin F 3rd, Conner W. Adenocarcinoma of the endometrium: survival comparisons of patients with and without pelvic node sampling. Gynecol Oncol. 1995; 56: 29-33. https://doi.org/10.1006/gyno.1995.1005.

3. Fanning J. Long-term survival of intermediate risk endometrial cancer (stage IG3, IC, II) treated with full lymphadenectomy and brachytherapy without teletherapy. Gynecol Oncol. 2001; 82: 371-4. https://doi.org/10.1006/ gyno.2001.6276.

4. Takeshima N, Hirai Y, Tanaka N, Yamawaki T, Yamauchi K, Hasumi K. Pelvic lymph node metastasis in endometrial cancer with no myometrial invasion. Obstet Gynecol. 1996; 88: 280-2. https://doi.org/10.1016/0029-7844(96)00115-9.

5. Larson DM, Broste SK, Krawisz BR. Surgery without radiotherapy for primary treatment of endometrial cancer. Obstet Gynecol. 1998; 91: 355-9.

6. Cragun JM, Havrilesky LJ, Calingaert B, Synan I, Secord AA, Soper JT, Clarke-Pearson DL, Berchuck A. Retrospective analysis of selective lymphadenectomy in apparent early-stage endometrial cancer. J Clin Oncol. 2005; 23: 3668-75. https://doi.org/10.1200/jco.2005.04.144.

7. Trimble EL, Kosary C, Park RC. Lymph node sampling and survival in endometrial cancer. Gynecol Oncol. 1998; 71: 340-3. https://doi.org/10.1006/gyno.1998.5254.

8. Ayhan A, Tuncer R, Tuncer ZS, Yüce K, Küçükali T. Correlation between clinical and histopathologic risk factors and lymph node metastases in early endometrial cancer (a 
multivariate analysis of 183 cases). Int J Gynecol Cancer. 1994; 4: 306-9.

9. Yenen MC, Dilek S, Dede M, Goktolga U, Deveci MS, Aydogu T. Pelvic-paraaortic lymphadenectomy in clinical Stage I endometrial adenocarcinoma: a multicenter study. Eur J Gynaecol Oncol. 2003; 24: 327-9.

10. Lo KW, Cheung TH, Yu MY, Yim SF, Chung TK. The value of pelvic and para-aortic lymphadenectomy in endometrial cancer to avoid unnecessary radiotherapy. Int J Gynecol Cancer. 2003; 13: 863-9.

11. Tanaka T, Terai Y, Ono YJ, Fujiwara S, Tanaka Y, Sasaki H, Tsunetoh S, Kanemura M, Yamamoto K, Yamada T, Ohmichi M. Preoperative MRI and intraoperative frozen section diagnosis of myometrial invasion in patients with endometrial cancer. Int J Gynecol Cancer. 2015; 25: 879-83. https://doi.org/10.1097/igc.0000000000000470.

12. Rechichi G, Galimberti S, Signorelli M, Franzesi CT, Perego P, Valsecchi MG, Sironi S. Endometrial cancer: correlation of apparent diffusion coefficient with tumor grade, depth of myometrial invasion, and presence of lymph node metastases. AJR Am J Roentgenol. 2011; 197: 256-62. https://doi.org/10.2214/ajr.10.5584.

13. Bharwani N, Miquel ME, Sahdev A, Narayanan P, Malietzis G, Reznek RH, Rockall AG. Diffusion-weighted imaging in the assessment of tumour grade in endometrial cancer. Br J Radiol. 2011; 84: 997-1004. https://doi.org/10.1259/ bjr/14980811.

14. Kishimoto K, Tajima S, Maeda I, Takagi M, Ueno T, Suzuki N, Nakajima Y. Endometrial cancer: correlation of apparent diffusion coefficient (ADC) with tumor cellularity and tumor grade. Acta Radiol. 2016; 57: 1021-8. https://doi. org/10.1177/0284185115612249.

15. Woo S, Cho JY, Kim SY, Kim SH. Histogram analysis of apparent diffusion coefficient map of diffusion-weighted MRI in endometrial cancer: a preliminary correlation study with histological grade. Acta Radiol. 2014; 55: 1270-7. https://doi.org/10.1177/0284185113514967.

16. Nougaret S, Reinhold C, Alsharif SS, Addley H, Arceneau J, Molinari N, Guiu B, Sala E. Endometrial Cancer: Combined MR Volumetry and Diffusion-weighted Imaging for Assessment of Myometrial and Lymphovascular Invasion and Tumor Grade. Radiology. 2015; 276: 797-808. https:// doi.org/10.1148/radiol.15141212.

17. Yan B, Zhao T, Liang X, Niu C, Ding C. Can the apparent diffusion coefficient differentiate the grade of endometrioid adenocarcinoma and the histological subtype of endometrial cancer? Acta Radiol. 2018; 59:363-370. https://doi. org/10.1177/0284185117716198.

18. Karabagli P, Ugras S, Yilmaz BS, Celik C. The evaluation of reliability and contribution of frozen section pathology to staging endometrioid adenocarcinomas. Arch Gynecol Obstet. 2015; 292: 391-7. https://doi.org/10.1007/ s00404-015-3621-5.

19. Stephan JM, Hansen J, Samuelson M, McDonald M, Chin Y, Bender D, Reyes HD, Button A, Goodheart MJ. Intra-operative frozen section results reliably predict final pathology in endometrial cancer. Gynecol Oncol. 2014; 133: 499-505. https://doi.org/10.1016/j.ygyno.2014.03.569. 